\title{
Introductory Remarks: Reflections on the Reasons for Building Accelerators
}

\author{
Andrew M. Sessler \\ Lawrence Berkeley Laboratory \\ Berkeley, CA 94720
}

April 8, 2002

\section{Introduction}

This is a good occasion, just when Fermilab is considering whether or not to build a new machine, and just when KEK/JAERI has initiated a new machine, to look back in history and review the reasons given -- even believed by the proponents -- for constructing a new accelerator. And then, of course, look at what really happened.

Now there is a fair amount of history, more than 80 years of it, as accelerators go back to the 20 's. Perhaps it is useful to categorize accelerators into groups. The first might be Energy Frontier Machines. Now that, at first sight, might not be relevant, but it is. For a driver can be used to make neutrino beams of high energy and, of course, become the driver for a neutrino factory, and some day, perhaps, for a muon collider. Plenty of frontier things.

The second category might be Special Purpose Machines, such as high intensity machines. Many of these are not in high energy physics, such as spallation sources, cyclotrons for nuclear physics, and medical accelerators. The relevance to this 
Workshop is obvious. A third category might be Machines for Advancing the Accelerator Art, where, once again, the relevance is obvious.

There are machines built for political reasons. This is not a disparagement at all, for some of our most productive accelerators were built primarily for political reasons, such as building --- or even maintaining --- infra-structure in a country or a laboratory. I will mention the political/sociological motives as we go along through the three categories that are inclusive of all accelerators.

Now, there have been a great many accelerators, even in high energy physics, so I can't be exhaustive here (I may be in some other venue). So I shall just pick a few to give you what I hope will be a balanced historical view.

\section{Accelerators on the Energy Frontier}

Consider, first, Accelerators on the Energy Frontier. The very earliest accelerators were, of course, in this category. I am thinking of the electrostatic machines that were constructed for, and did achieve, artificial nuclear reactions. Then, of course, there were, in the 30's, the cyclotrons. (And that was it, electrostatic machines and cyclotrons, until after WWII !) The cyclotrons were built with no deeper thought, as far as I can see, except to get to ever-higher energy. But the intuition was right: no one dreamed of the wealth of physics they would be opened up: nuclear isotopes, the discovery of tritium, and then all the transuranics (including plutonium and all that followed in reactors and bombs), nuclear medicine, and, then, just after WWII, the production of pions. 
The Cosmotron was the first accelerator built at Brookhaven, simply to get to even higher energies than previously achieved. It rather quickly led TD Lee and CN Yang to interpret the data on weak decays as violations of parity; a result no one envisioned when the device was initiated. (Nobel Prize)

The Bevatron was a political deal (the proposal was one page long) as was the AGS. (Something for Berkeley on the West Coast and something for Brookhaven on the East Coast. In those days the AEC didn't see the middle of the country.) The Bevatron was built to discover, and it did, the anti-proton, but its real contribution was the discovery of "resonances", which weren't even dreamed of when it was proposed and constructed. (Two Nobel Prizes)The AGS was used to discover two neutrinos, time reversal non-invariance, and the J/Psi particle, three Nobel Prizes and three phenomena not even dreamed of when the AGS was constructed.

Electron linacs were developed after WWII and electron scattering off nuclei allowed the first precision determination of the nuclear radius and charge distribution. Subsequently, and in good measure because of this success, SLAC was constructed, and the most important discovery on that machine was the disclosure of the parton structure of the nucleon, a completely unexpected result. (Nobel Prize)

Electron storage rings were built to study QED at everhigher energies, i.e., at ever-smaller distances. But no one expected the J/Psi particle and the November revolution. (Nobel Prize)

I could tell you about some more modern machines, but I believe you can see, already, that the really interesting frontier 
machines were machines with which unexpected phenomena were disclosed. Of course it didn't always turn out that frontier machines produced unexpected phenomena. I can think of PEP, PETRA, Tristan, all built in the enthusiasm following the November revolution and all producing minimal physics. Some machines were built for strong political reasons, such as the PS to bring Europe into high-energy physics, or the Beijing ring for China, or the proton accelerator at KEK for Japan. Essentially all served the designated purpose, and, as such, were very important to the sociology of high-energy physics; some also produced very important physics.

I believe one can be rather confident that a driver at Fermilab, or the driver underway at KEK/JAERI will be employed to study frontier physics. Whether, or not, significant discoveries will be made with these drivers is, of course, beyond me, but with the first signs of physics beyond the Standard model, now in hand, it would take a very pessimistic person to believe that little will come of these machines.

\section{Special Purpose Machines}

Turning our attention to Special Purpose Machines, the largest number of these is, of course, those produced for medical purposes; both the production of isotopes for diagnostic purposes and the therapy of cancer. One third of the patients in hospitals have a procedure involving nuclear isotopes, which, often, are made by accelerators. In regard to therapy, 1.75 M patients a year in the US receive accelerator treatments. Counting just electron machines, there are more than 3000 , which at about $1 \mathrm{M} \$$ apiece, is an investment of 3 $\mathrm{B} \$$. This is the major spin-off of the accelerator art to the economy. Besides these two accelerator spin-offs of highenergy physics, there are the large superconducting magnets 
used in MRI. These three items are, perhaps, the major spinoffs of high-energy physics.

Then, of course, if we go up in energy, there are the electron synchrotrons. The last I looked there were some 70 around the world. The big ones are the ESR in Europe, the APS in the US, and Spring 8 in Japan. Major investments; the APS must be about $800 \mathrm{M} \$$. The impact on society is usually indirect - not quite so direct as treating a cancer patient - but very real in the long term.

Then there are the spallation neutron sources, the small ones that already operate, and the very large ones under construction in the US and in Japan. (With Europe probably to follow.) The SNS is, to set the scale, a 1.3 B\$ device.

Still going up in energy there are the B-Factories.

Certainly built with a very clear, and well-defined, purpose in mind. These devices, both the ones at KEK and at SLAC, have performed even better than promised and are well on the way to making significant discoveries about time reversal noninvariance.

In sum, one sees that the machines built for a special purpose have, in all cases that I am aware of, worked as expected. I believe that also will be the case for the JHF and the proposed booster here.

\section{Machines for Advancing the Accelerator Art}

All new concepts, when first developed experimentally went through this phase. In the old days it was easy. The first cyclotron was 5 inches across. The first test of the phase focusing concept was on a $300 \mathrm{MeV}$ electron synchrotron, the 
first linacs were small devices, the first test of $A G$ was on a Cornell electron synchrotron.

Three rather large tests come to mind and illustrate the need -- or the perceived need at the time -- for extensive (and expensive) testing. The first was the concept of colliding proton beams. The theory was developed, primarily here in the Midwest, various aspects (such as rf stacking) were tested in the Midwest, and then the concept was taken seriously by CERN and the long-term stability was tested on a very low energy electron ring. But complete test required the ISR, which was probably, the most expensive accelerator development device ever made. Very little physics was done on the ISR (in part because the interesting events were at 90 degrees, not forward, and only in the last experiments were physicists even looking at such events).

The second was the concept of stochastic cooling, which didn't require a high-energy machine for testing. The testing was done on ICE, which was, in itself, non-trivial in size and cost, and very complex indeed. The resulting physics, the discovery of the $\mathrm{Z}$ and the $\mathrm{W}$, were great events.

The third example is linear colliders of electrons. Here a very large device, the SLC, was built and I remember how it was greeted by skepticism on the part of the high-energy community (as was also true for the other two examples). In fact the SLC was originally only an accelerator project, but later Z-physics was done on the device (as well as, of course, on LEP).

The accelerator art has only been able to move forward, in very significant steps, with major tests. Sure, many advances were made in bench studies, or relatively minor 
studies (such as the pretzel scheme studies at Cornell, or rf Qs). For muons we are going to require rather major tests, such as the targetry work at Brookhaven, MUCOOL here, and the International Cooling Experiment, MICE. Eventually, however, as with the ISR, it all has to be put together and having a driver at Fermilab, or using he driver at KEK/JAERI, will be the necessary front end for a neutrino factory, and eventually, a muon collider. I am confident that the accelerator art will continue to take bold steps; for if it doesn't that will mean the end of high-energy physics, at least as we have known it for he last 80 years, and I don't think any of us are envisioning that. But we need more than "vision"; we need action to prevent the demise of high-energy physics.

\section{Reprieve}

In this session, and really for most of this week, we shall look in detail, and explore, the accelerator aspects of high intensity and high brightness hadron beams. The technical issues are many-fold and quite fascinating. I hope the result of all these discussions of multitudinous effects at a variety of machines will be renewed enthusiasm for moving ahead here at Fermilab. For there are good reasons for moving ahead; at least as good as the reasons given in the past for constructing new machines. And, think, without that past optimism we would be in a very different place today. 
